\title{
Dependence of Intense Geomagnetic Storms on the Interplanetary Field / Plasma Parameters during Solar Cycle 23 \& 24
}

\author{
Seema Pande ${ }^{1}$, Mahesh Chandra Mathpal ${ }^{2}$, Bimal Pande ${ }^{3}$ \\ Assistant Professor, Department of Physics, DSB Campus Kumaun University, Nainital, India ${ }^{1,3}$ \\ Junior Research Fellow, Department of Physics, DSB Campus Kumaun University, Nainital, India ${ }^{2}$
}

\begin{abstract}
We present a statistical analysis of the peak values of geomagnetic activity indices ( $\mathrm{Kp}$, ap, AE and Dst ) with the interplanetary plasma and field parameters $(\mathrm{T}, \mathrm{D}, \mathrm{V}, \mathrm{P}$, and $\mathrm{Bt}, \mathrm{Bz}, \mathrm{E}$, and $\mathrm{B}$ ) along with their product functions $\left(\mathrm{BV}, \mathrm{BzV}\right.$ and $\left.\mathrm{B}^{2} \mathrm{~V}\right)$ during major geomagnetic storms. We find that peak values of Dst, $\mathrm{Kp}$, ap, and $\mathrm{AE}$ are in good correlation with $\mathrm{E}, \mathrm{V}, \mathrm{BV}, \mathrm{BzV}, \mathrm{B}^{2} \mathrm{~V}, \mathrm{Bt}, \mathrm{Bz}$. We have obtained specifically high values of correlation between Dst and product functions of plasma parameters $\left(\mathrm{R}=-0.75\right.$ for $\mathrm{BV}, \mathrm{R}=0.78$ for $\mathrm{BzV}$ and $\mathrm{R}=-0.77$ for $\mathrm{B}^{2} \mathrm{~V}$ ). This study provides statistical prove that occurrence of intense GMSs depends mainly upon magnetic field, velocity and their product functions $\left(\mathrm{BV}, \mathrm{BzV}\right.$ and $\left.\mathrm{B}^{2} \mathrm{~V}\right)$. Therefore these parameters may act as reliable indicators for predicting GMSs and their strength. We have also analysed the GMSs data during different phases of the solar cycles $23 \& 24$ and concluded that CMEs are more important drivers of GMSs during the maximum phase of solar cycle while CIR are more significant drivers of GMSs during the decay phase.
\end{abstract}

Keywords: Geomagnetic Storms, interplanetary field parameters, interplanetary plasma parameters, Geomagnetic Indices.

\section{INTRODUCTION}

The Sun displays manifestations of periodic magnetic phenomena in solar atmosphere called solar activity features. The 11 year time period in which solar activity varies is called solar cycle. The variation in solar activity is attributed to the variation in magnetosphere-ionosphere system. Solar wind and coronal mass ejection (CME), which originate from the Sun, are directly connected to magnetic field of the Earth's magnetosphere, This process is known as magnetic reconnection. This magnetic reconnection produces a disturbance in the magnetosphere-ionosphere system called geomagnetic storm. [1,2] stated that this disturbance gives rise to several changes in interplanetary and terrestrial environment. The solar wind at Earth orbit has mean density of about $4 \mathrm{~cm}^{-3}$, mean velocity of about $400 \mathrm{kms}^{-1}$ and mean interplanetary magnetic field (IMF) magnitude of $5 \mathrm{nT}$. The average direction of IMF along the parker spiral in the ecliptic plane is at an angle of $45^{\circ}$ from the redial direction [3].GMSs are driven by magnetic reconnection between IMF and terrestrial magnetic field. As the dipole is close to perpendicular to the ecliptic plane which depends on southward component of the IMF, the reconnection rate is proportional to the $\mathrm{Y}$ - component of the motional electric field $(\mathbf{E}=$ Vsw $\times$ BIMF $)$ of the solar wind[4].Coronal mass ejection (CMEs) expel vast clouds of solar magnetic flux and plasma into interplanetary space. The interplanetary space formed by the CME propagates from the Sun, often at high velocity[5].The coherent magnetic field structure, the strong varying field and plasma density in the sheath region preceding the ICME proper, the fast solar wind speed as well as the interplanetary shock itself are all effective drivers of geomagnetic activities [6].

Solar wind \& CMEs are the main drivers of geomagnetic storms. High speed solar wind streams originate from low latitudes coronal holes, encounter the Earth \& give rise to large fluctuations in IMF Bz \& solar wind velocity. These are effective drivers of medium level activity in the high latitude magnetosphere [7].The storms associated with them are called CME driven geomagnetic storms(GMSs). When the high speed solar wind that originates from coronal holes decelerates and interacts with magnetosphere, its leads to a compression of the plasma and magnetic fields forming corotating interaction region (CIR) $[8,9]$.The storms which are associated with such interaction are called CIR driven GMSs. The CIRs driven GMSs exhibit fast shock and continuous strongly southward IMF Bz and thus depict only moderate geomagnetic activity [10,11]. CIR being associated with the coronal hole structure, also exhibits 27 day periodicity [12].

Indices used to describe the variation in geomagnetic field are called geomagnetic indices. Dst,kp,ap,A.E are the most commonly used geomagnetic activity indices [13] .GMSs are classified on the bases of Dst

Index as: Intense storms (Dst $\leq-100 \mathrm{nT})$ moderate storms $(-100 \mathrm{nT}<$ Dst $<-50 \mathrm{nT}) \&$ weak storms $($ Dst $>-50 \mathrm{nT})[2]$. 
There are a number of statistical studies which define relationship between geomagnetic indices and interplanetary field parameters. These studies are very important to predict space weather. A statistical study of Kp and ap index with interplanetary field parameters represented the planetary intensity of magnetic activity at subauroral latitude [14, 15, 16]. $[17,18,19,20]$ studied the relationship of intense GMSs with intense IMF and its southern components for a long time (> 3hour).[21] studied the relationship between Dst index solar wind speed and product of southern components $\mathrm{Bz}$ of IMF and wind speed V for several events during 1973-2003.This study found that product of southern components and speed investigates the occurrence of moderate and strong GMSs.[18] studied the relationship between 64 GMSs event of Dst index (Dst $\leq-85 \mathrm{nT}$ ) with southern components Bz of IMF during period of 1997-2002 and found that $75 \%$ of peak value of $\mathrm{Bz}$ is entire event at peak value of Dst. Coordinated data analysis workshop (CDAW) held at Georgemason university Fairfax Viginia in march 2005 and second workshop held at Florida institute of technology Melbource Folirda in March 2007 focused on the role of CMEs or CIR as the source of geomagnetic storms. These workshops decided the 88 major geomagnetic storms events during the period of 1996 to 2005 and explained how to will decide the solar and interplanetary sources of GMSs.[22] represented a statistical study of interplanetary field parameters with intense geomagnetic storm (Dst $\leq-100 \mathrm{nT}$ ) of cycle 23 (1996-2006).However, there is a strong need to study the dependence of interplanetary field parameters and plasma field parameters with intense geomagnetic indices (GI) for a large data set.

\section{DATA ANALYSIS AND STATISTICAL}

\section{Study:}

In the present work we have attempted a detailed study of intense geomagnetic storms (Dst $\leq-100 \mathrm{nT})$ and analysed the dependence of intense geomagnetic storms on interplanetary field parameters(Bt,Bz,E,B) and plasma parameters(T,V,D,P,) for a period of 20 years during 1996 to 2016 ( solar cycle 23 \& 24). During this period 109 intense geomagnetic storms appeared .Out of them 92 events occurred in cycle 23 and 16 events occurred in cycle 24 . Solar cycle 24 contains less number of GMSs since there is a significant drop in density, magnetic field, total pressure and Alfven wave speed in the inner Helosphere [23]. The total number of events during this period is classified in two groups- $95 \mathrm{CME}$ driven events and $14 \mathrm{CIR}$ driven events. The data sets for Geomagnetic indices and interplanetary field and plasma parameters data were taken from OMNI website at: http://swdc.kugi.kyoto.u.ac.jp/dstdir

For all the 109 events the peak values of Geomagnetic indices (GIs),inter-planetary field parameters and plasma parameters are presented in table 1 . We have used a linear regression analysis $\mathrm{Y}=\mathrm{A}+\mathrm{BX}$ for these parameters where $\mathrm{Y}$ is peak value of GIs and $\mathrm{X}$ is interplanetary field /plasma parameters. We have studied the relationship between these parameter in different phases of solar cycle 23\& 24 namely rising phase (1996 -1999; 2008-2011) maximum phase (2000 - 2002; 2012 -2014) and decay phase (2003-2008; 2015-2016). We have also calculated correlation coefficient, average, median and standard deviation of peak values of various GIs and interplanetary /plasma field parameters in different phases of solar cycle $23 \& 24$ as well as for total period 1996 - 2016.

\section{ANALYSIS AND RESULTS}

\subsection{Relationship between peak values of geomagnetic activity indices and interplanetary field/Plasma parameters:}

To understand the dependence of intense geomagnetic storms on the interplanetary field and plasma parameters during 1996-2016 (cycle 23 \& 24), we have represented a statistical correlative study between GIs and interplanetary field/plasma parameters and their functions $\left(\mathrm{BV}, \mathrm{BzV}, \mathrm{B}^{2} \mathrm{~V}\right)$. Figure 1-figure 4 represent the scatter plots of GIs, interplanetary field and plasma parameters as well as their product functions $\left(\mathrm{BV}, \mathrm{BzV}, \mathrm{B}^{2} \mathrm{~V}\right)$. The linear regression equation and their correlation coefficient are given in all plots. The correlation coefficients of GIs with interplanetary field/plasma parameters and their product functions $\left(\mathrm{BV}, \mathrm{BzV}, \mathrm{B}^{2} \mathrm{~V}\right)$ in different phases of solar cycle 23 \& 24 and during whole period 1996-2016 ( cycle 23-24) are represented in table 2. It can be seen from fig.1.that Dst shows good correlation with $\mathrm{Bt}(\mathrm{R}=-0.79), \mathrm{Bz}(\mathrm{R}=0.75), \mathrm{E}(\mathrm{R}=$

$0.72), \mathrm{BV}(\mathrm{R}=-0.75), \mathrm{BzV}(\mathrm{R}=0.73), \mathrm{B}^{2} \mathrm{~V}(\mathrm{R}=0.77)$ and moderate correlation with $\sigma \mathrm{B}(\mathrm{R}=-0.54)$ and $\mathrm{V}(\mathrm{R}=-0.53)$ whereas it shows weak correlation with $\mathrm{T}(\mathrm{R}=-0.34), \mathrm{D}(\mathrm{R}=0.13), \mathrm{P}(0.43)$ and $\beta($ beta $)(\mathrm{R}=-.026)$. Fig.2. indicates that $\mathrm{Kp}$ index shows good correlation with $\mathrm{Bt}(\mathrm{R}=0.72), \mathrm{V}(\mathrm{R}=0: 65), \mathrm{BV}(\mathrm{R}=0.74) \mathrm{BzV}(\mathrm{R}=-0.66), \mathrm{B}^{2} \mathrm{~V}(\mathrm{R}=-0.66)$, moderately correlated with $\mathrm{Bz}(\mathrm{R}=-0.60), \sigma \mathrm{B}(\mathrm{R}=-0.51), \mathrm{E}(\mathrm{R}=0.55), \mathrm{T}(\mathrm{R}=0.51), \mathrm{P}(\mathrm{R}=0.57)$ and this index is weakly correlated with $\mathrm{D}(\mathrm{R}=0.24), \beta$ (beta) $(\mathrm{R}=0.08)$. From figure 3 , We can see that ap shows good correlation with $\mathrm{Bt}(\mathrm{R}=$ $0.78), \mathrm{Bz}(\mathrm{R}=-0.66), \mathrm{E}(\mathrm{R}=0.73), \mathrm{V}(\mathrm{R}=0.72), \mathrm{BV}(\mathrm{R}=0.83), \mathrm{BzV}(\mathrm{R}=0.77), \mathrm{B}^{2} \mathrm{~V}(\mathrm{R}=0.77)$, moderate correlation with $\sigma B(\operatorname{sigma}(B))(R=0.54), T(R=0.54), P(R=0.56)$ while weak correlation with $\mathrm{D}(\mathrm{R}=0.17), \& \beta($ beta $)(\mathrm{R}=0.04)$.It is clear from fig 4. that A.E shows good correlation with $\mathrm{Bt}(\mathrm{R}=0.63), \mathrm{V}(\mathrm{R}=0.63), \mathrm{BV}(\mathrm{R}=0.68)$ and moderate correlation with $\mathrm{Bz}(\mathrm{R}=-0.50), \sigma(\mathrm{B})(\mathrm{R}=0.52), \mathrm{E}(\mathrm{R}=0.54), \mathrm{T}(\mathrm{R}=0.54), \mathrm{P}(\mathrm{R}=0.55), \mathrm{BzV}(\mathrm{R}=0.58), \mathrm{B} 2 \mathrm{~V}(\mathrm{R}=0: 60)$ whereas it is weakly correlated with $\mathrm{D}(\mathrm{R}=0.23), \beta$ (beta) $(\mathrm{R}=0.12)$. This indicates that GIs are in good correlation with 
interplanetary field and plasma parameters except density (D) and plasma $\beta$ parameter. To understand the mechanism in a better way we have examined the correlation of peak values of different indices with various product functions $(\mathrm{BV}, \mathrm{BzV}, \mathrm{B} 2 \mathrm{~V})$. When we analyse this data in different phases of cycle $23 \& 24$, we see that GIs show good correlation with interplanetary field/ plasma parameters and specifically high correlation with their product function. However we get weak correlation with density (D), pressure (P) and Plasma $\beta$ (beta) parameters. GIs are highly correlated with these product functions which indicate that variation in the values of these product functions may serve as reliable indicator of geomagnetic activities thereby allowing us to predict the strength of GMSs.

\subsection{Average, Median, Standard deviation analysis of various geomagnetic indices, interplanetary field and plasma parameters:}

Average, Median, Standard deviation analysis of various geomagnetic indices, interplanetary field and plasma parameters during cycle23 \& 24(1996-2016) have been represented in table 3. We have presented a comparison of average and median of GIs, interplanetary field and plasma parameters and their product functions during the rising, maximum and decay phase of solar cycles 23-24. From table 3, it is clear that averages of GIs, interplanetary field and plasma parameters increase during the decay phase as compared to the rising and maximum phase. Though the number of events in decay phase is less ( 29 events) but there intensity is relatively high (Dst index reaching up to $-442 \mathrm{nT}$ in solar cycle $23 \&$ up to $-223 \mathrm{nT}$ in solar cycle 24 ) as compared to the events of rising and maximum phase. This accounts for the higher average values in decay phase.

\section{CONCLUSION}

The present study intends to examine the dependence of GIs on interplanetary field and plasma parameters. The whole study has been focused upon analyzing the relationship among peak values of GIs, interplanetary field and plasma parameters. We have found that GIs exhibit good correlations with interplanetary field and plasma parameters, which indicates that these parameters play an important role to determine the strength of storms and sub storms.

These may also prove vital in making prediction about geomagnetic storms and sub-storms and their strength. We have also presented a correlated study of various GIs with interplanetary field and plasma parameters. Average, median and standard deviation analysis is also done for different phases of cycle $23 \& 24$ throughout the 20 -year period. These analyses indicate that geomagnetic storms immensely affect the magnetospheric environment and occurrence of intense geomagnetic storms depends on the variation in the values of interplanetary field and plasma parameters. Since most of the events 95(87:2\% of total event) appear in CME driven and 14 events (12:8\% of total event) appear in CIR driven hence CME are more geoeffective driver than CIR. The phase analysis (rising phase, maximum phase and decay phase) for the two solar cycles $23 \& 24$ indicates that most of the events (45:9\% of total events) occurred in maximum phase which implies that maximum phase is more geoeffective than the other phases. However when we made the phase analysis separately, we found that maximum number of events occurred in the maximum phase(47:4\% ) for CME driven events while maximum number of events occurred in the decay phase $(50 \%)$ for CIR driven events. This indicates that CMEs are more important drivers of GMSs during the maximum phase of solar cycle while CIR become more significant drivers of GMSs during the decay phase of solar cycle.

Solar wind contains southward components of magnetic field and velocity by which GMSs originated in the magnetosphere[5, 6].Our statistical study indicate that GIs shows good correlation (positive as well as negative) with magnetic field, velocity and their product functions $(\mathrm{BV}, \mathrm{BzV}, \mathrm{B} 2 \mathrm{~V})$. We also found that GIs always show correlation coefficient $\geq 0.70$ with their product functions either in the whole period or different phases of solar cycle $23 \& 24$.A possible reason behind this may be that magnetic field and velocity are factors which produce the geomagnetic storms in magnetosphere-ionosphere system. Combined effect of magnetic field and velocity must be much better than individual magnetic field and velocity. Hence our study gives a statistical prove that the occurrence of intense geomagnetic storms depends on the interplanetary field and plasma parameters.

\section{REFERENCES}

[1] Echer, E., Gonzalez, W.D., Tsurutani, B.T.,Gonzalez, A.L.C., 2008. JGR 113, A05221

[2] . Gonzalez, W.D., Joselyn, J.A., Kamide, Y.,Kroehl, H.W., Rostoker, G., Tsurutani, B.T.,

Vasyliunas, V.M., 1994. JGR 99, 5771.

[3] Hundhausen,A.J.,1972, Coronal Expansion and Solar Wind, vol 5 of hysics and chemistry in space springer, Berlin,Germany; NewYorks,U.S.A 2

[4] Vasyliunas, V.M., 1975, "Theoretical Models of magnetic field line mergign.I",Rev.Geophys.Space phys.,13,303-336 2

[5] Schwenn,R.,2006,"Space Weather: The Solar Perspective",Live Rev. Solar Phys.,3 lrsp 2006-2.

[6] Farrugia,C.J., Burlaga,L.F., Lepping,R.P.,Tsurutani,B.T.,Gonzalez,W.D.,Kamide,Y., Arballo,J.k., 1997, vol.98 of geophysical monograph,pp.91106, American Geophysical Union Washington,U.S.A.2

[7] Tsurutani, B.T., Gonzalez, W.D., 1987, SpaceSci., 35, 405-412. 


\section{International Advanced Research Journal in Science, Engineering and Technology ISO 3297:2007 Certified}

Vol. 4, Issue 7, July 2017

[8]Crooker,N.U.,Gosling,J.T.,Bothmer,V.Forsyth,R.J.,Gazis,P.R.,Hewish,A.,Horbury,T.S.,Intriligator,D.S.,Jokipii,J.R.,Kota,J.,Lazarus,A.J.,Lee,M.A .1,Lucek,E.,Marsch,E.,Posner,A.,Richardson,I.G.,Roelof,E.C.,Schmidt,J.M.,Siscoe,G.L.,Tsurutani,B.T.,Wimmer-Schweingruber,R.F.,1999., Space sci.Rev.,89,179-220 2

[9] Zhang, J., Richardson, I.G., Webb, D.F.,Gopalswamy, N., Huttunen, E., Kasper, J.C.,Nitta, N.V., Poomvises, W., Thompson, B.J.,Wu, C.-C., Yashiro, S., Zhukov, A.N., 2007.JGR 112, A10102

[10] Alves,M.V., Echor, E., Gonzalez,W.D., 2006,J.Geophys.Res., 1112

[11] Borovsky,J.K.,Denton,M.H.,2006, J Geo-phys.Res.Lett., 24,927-929 7.4

[12] Schwenn,R.,1990,vol 1-vol 20 physics and chemistry in space ,pp.99-181,Springer, Berlin,Germany; New york,U.S.A.2

[13] Rostoker, G., 1972. Rv GSP 10, 935

[14] Badruddin 1998. P\& SS 46, 1015

[15] Crooker, N.U., Gringauz, K.I., 1993, JGR 98,59.

[16] Dwivedi, V.C., Triwari, D.P., Agrawal, S.P.,2009. JGR 114, A05108

[17] Gonzalez, W.D., Tsurutani, B.T., 1987. P\& SS 35, 1101

[18] Gonzalez, W.D., Echer, E., 2005. GeoRL 32,L18103

[19] Echer, E., Gonzalez, W.D., Tsurutani, B.T., Gonzalez, A.L.C., 2008. JGR 113, A05221

[20] Kane, R.P., 2010. P\& SS 58, 3925

[21] Kane, R.P., 2005. JGR 110, A02213

[22] Joshi, N.C., Bankoti, N.S., Pande, S., Pande.B., Pandey, K.,2011. New Astronomy, 16,366-385

[23] Gopalswamy, N., Akiyama.S., yashiro.S., Xie.H., Makela.P., Michalek.G.,14th International Ionospheric Effects Symposium on 'Bridging the gap between applications and research involving ionospheric and space weather disciplines' May 12-14, 2015, Alexandria, VA

\begin{tabular}{|c|c|c|c|c|c|c|c|c|c|c|c|c|c|c|c|c|c|c|}
\hline $\begin{array}{l}Z \\
\dot{n}\end{array}$ & 尽 & 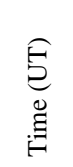 & $\underbrace{\stackrel{E}{\Xi}}_{\tilde{\omega}}$ & 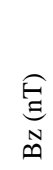 & 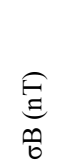 & $\begin{array}{l}\stackrel{\mathcal{J}}{\dot{\Xi}} \\
\underset{\Xi}{\Xi} \\
\Xi\end{array}$ & $\underset{E}{E}$ & $\begin{array}{l}\overbrace{1}^{\tilde{c}} \\
0 \\
0\end{array}$ & 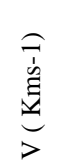 & $\underbrace{\overparen{\Xi}}_{0}$ & $\stackrel{\pi}{0}$ & 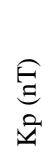 & 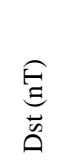 & $\underset{⿱ 乛 ⿻ 上 丨 匕}{E}$ & $\begin{array}{l}\underset{\mathrm{E}}{\Xi} \\
\frac{2}{4}\end{array}$ & 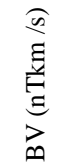 & 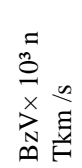 & 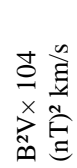 \\
\hline 1 & $21 / 04 / 1997$ & $23: 00$ & 13.9 & -7.7 & 8 & 4.01 & 113138 & 30.5 & 441 & 7.97 & 13.12 & 5.3 & -107 & 917 & 56 & 6.13 & -3.4 & 8.52 \\
\hline 2 & $15 / 05 / 1997$ & $13: 00$ & 25.2 & -24 & 13.2 & 10.23 & 341122 & 29.8 & 524 & 10.2 & 18.4 & 6.7 & -115 & 865 & 111 & 13.2 & -12.73 & 33.28 \\
\hline 3 & 10/11/1997 & $4: 00$ & 13.4 & -10 & 7.1 & 4.51 & 104825 & 30.2 & 449 & 11.8 & 2.67 & 6.3 & -130 & 1043 & 94 & 6.02 & -4.58 & 8.06 \\
\hline 4 & $07 / 11 / 1997$ & $5: 00$ & 17.9 & -13 & 11.9 & 5.62 & 374718 & 23.4 & 468 & 9.98 & 4.17 & 7 & -110 & 981 & 132 & 8.38 & -5.85 & 15 \\
\hline 5 & 23/11/1997 & $7: 00$ & 26.3 & -13 & 18.9 & 6.46 & 415513 & 32.4 & 591 & 15.8 & 5.01 & 7.3 & -108 & 985 & 154 & 15.54 & -7.56 & 40.88 \\
\hline 6 & $18 / 02 / 1998$ & $1: 00$ & 21.1 & -13 & 11.4 & 6.18 & 298693 & 24.4 & 478 & 8.24 & 5.9 & 6.7 & -100 & 1210 & 111 & 10.09 & -6.17 & 21.28 \\
\hline 7 & $04 / 05 / 1998$ & $6: 00$ & 38 & -20 & 18.8 & 23.82 & $1.00 \mathrm{E}+06$ & 26 & 833 & 36.9 & 3.43 & 8.7 & -205 & 1443 & 300 & 31.65 & -16.24 & 120.29 \\
\hline 8 & $26 / 06 / 1998$ & $5: 00$ & 17.4 & -13 & 11 & 6.04 & 106347 & 20.7 & 496 & 8.92 & 6.78 & 6.3 & -101 & 1234 & 94 & 8.63 & -6.55 & 15.02 \\
\hline 9 & $06 / 08 / 1998$ & $12: 00$ & 20.3 & -18 & 9.4 & 8.26 & 128854 & 39.5 & 428 & 11.3 & 4.94 & 7.3 & -138 & 971 & 154 & 8.69 & -7.49 & 17.64 \\
\hline 12 & $25 / 09 / 1998$ & $10: 00$ & 25.4 & -18 & 16.6 & 13.66 & 907650 & 14.6 & 839 & 12.6 & 3.04 & 8.3 & -207 & 1432 & 236 & 21.31 & -15.44 & 54.13 \\
\hline 13 & $19 / 10 / 1998$ & $16: 00$ & 26.1 & -22 & 13.2 & 6.71 & 504349 & 65.3 & 698 & 21.9 & 6.47 & 6.7 & -112 & 1020 & 111 & 18.22 & -15.5 & 47.55 \\
\hline 14 & $08 / 11 / 1998$ & $7: 00$ & 34.7 & -12 & 8.9 & 12.15 & 331265 & 24.4 & 639 & 14.4 & 5.31 & 7.7 & -149 & $* * *$ & 179 & 22.17 & -7.41 & 76.94 \\
\hline 15 & $09 / 11 / 1998$ & $18: 00$ & 21.7 & -13 & 7.2 & 7.35 & 136799 & 23 & 522 & 12.5 & 10.43 & 6.7 & -142 & $* * *$ & 111 & 11.33 & -6.89 & 24.58 \\
\hline 16 & $13 / 11 / 1998$ & $22: 00$ & 20.9 & -18 & 12.6 & 7.13 & 534806 & 39.6 & 547 & 13.3 & 6.33 & 6 & -131 & $* * *$ & 80 & 11.43 & -9.63 & 23.89 \\
\hline 17 & $14 / 04 / 1999$ & $0: 00$ & 18.6 & -16 & 11.6 & 6.04 & 290371 & 30.9 & 593 & 10.92 & 3.51 & 7 & -112 & 994 & 132 & 11.03 & -9.31 & 20.52 \\
\hline 18 & $18 / 02 / 1999$ & $10: 00$ & 28.1 & -24 & 20 & 13.84 & 518339 & 13.7 & 673 & 10.7 & 2.99 & 6.7 & -123 & 1555 & 111 & 18.91 & -16.29 & 53.14 \\
\hline 19 & 23/09/1999 & $0: 00$ & 26.2 & -19 & 17.7 & 9.39 & 320171 & 46 & 602 & 18.6 & 2.73 & 8 & -173 & 928 & 207 & 15.77 & -11.14 & 41.32 \\
\hline 20 & $22 / 10 / 1999$ & $7: 00$ & 35.6 & -28 & 15.8 & 16.21 & 525759 & 49.8 & 678 & 27.5 & 3.06 & 8 & -237 & 1139 & 207 & 24.14 & -19.12 & 85.93 \\
\hline 21 & $13 / 11 / 1999$ & $23: 00$ & 11.7 & -11 & 8.7 & 5.39 & 155396 & 12.9 & 482 & 2.45 & 4.77 & 6.3 & -106 & 756 & 94 & 5.64 & -5.35 & 65.98 \\
\hline 22 & $12 / 02 / 2000$ & $12: 00$ & 19.4 & -15 & 14.2 & 9.56 & 413479 & 24.3 & 681 & 15.7 & 7.94 & 6.7 & -133 & 1124 & 111 & 13.21 & -10.21 & 25.63 \\
\hline 23 & $07 / 04 / 2000$ & $1: 00$ & 30.3 & -22 & 15 & 15.78 & 345986 & 33.4 & 625 & 20.3 & 4.19 & 8.7 & -288 & 1550 & 300 & 8.94 & -13.82 & 57.38 \\
\hline 24 & $16 / 07 / 2000$ & $1: 00$ & 50.6 & -49 & 33 & 51.38 & $2.00 \mathrm{E}+06$ & 20.6 & 1107 & 41.2 & 5.6 & 9 & -301 & 2023 & 400 & 56.01 & -54.56 & 28.34 \\
\hline 25 & $11 / 08 / 2000$ & $7: 00$ & 13.5 & -13 & 4.1 & 5.77 & 260592 & 11.7 & 441 & 4.23 & 0.79 & 5.7 & -106 & 1107 & 67 & 59.54 & -5.73 & 80.37 \\
\hline 26 & $12 / 08 / 2000$ & $10: 00$ & 33.3 & -26 & 22.7 & 17.33 & 463682 & 18.2 & 671 & 12.4 & 3.22 & 7.7 & -235 & 1724 & 179 & 22.37 & -17.72 & 74.4 \\
\hline 27 & $18 / 09 / 2000$ & $0: 00$ & 36.6 & -23 & 25.9 & 14.91 & 920518 & 32.8 & 839 & 25.5 & 2.51 & 8.3 & -201 & 1013 & 236 & 30.71 & -19.3 & 11.24 \\
\hline 28 & $05 / 10 / 2000$ & 14:00 & 25.8 & -25 & 16 & 9.55 & 261737 & 32.1 & 531 & 15.7 & 12.24 & 7.7 & -182 & 1456 & 179 & 13.69 & -13.06 & 35.35 \\
\hline 29 & $14 / 10 / 2000$ & $15: 00$ & 19.5 & -13 & 10.4 & 6.49 & 379120 & 29.2 & 584 & 11.1 & 3.52 & 6.7 & -107 & 1133 & 111 & 11.39 & -7.71 & 22.21 \\
\hline 32 & $29 / 11 / 2000$ & $14: 00$ & 13.7 & -12 & 7.2 & 5.57 & 250108 & 17.1 & 587 & 14 & 3.15 & 6.7 & -119 & 1076 & 11 & 8.04 & -7.04 & 11.01 \\
\hline 33 & $20 / 03 / 2001$ & $14: 00$ & 21.4 & -20 & 13 & 7.33 & 138018 & 23.5 & 490 & 9.87 & 4.43 & 7.3 & -149 & 1372 & 154 & 10.49 & -9.7 & 22.44 \\
\hline 34 & $31 / 03 / 2001$ & $9: 00$ & 46.3 & -46 & 41.8 & 30.62 & 704151 & 37.8 & 821 & 38.8 & 1.61 & 8.7 & -387 & 1524 & 300 & 38.01 & -38 & 175.99 \\
\hline 35 & $12 / 04 / 2001$ & $0: 00$ & 33.1 & -18 & 20.3 & 14.86 & 849339 & 24.7 & 832 & 24.5 & 4.31 & 8.3 & -271 & 1699 & 236 & 27.54 & -14.73 & 91.15 \\
\hline 36 & $18 / 04 / 2001$ & $7: 00$ & 22.7 & -13 & 12.3 & 9.72 & 393365 & 29.6 & 518 & 14.4 & 5.37 & 7.3 & -102 & 1753 & 154 & 11.76 & -6.83 & 26.69 \\
\hline 37 & $22 / 04 / 2001$ & $16: 00$ & 15.1 & -12 & 6.3 & 4.63 & 188.33 & 29.7 & 445 & 7.18 & 6.06 & 6.3 & -102 & 982 & 94 & 6.72 & -5.34 & 10.15 \\
\hline 38 & $17 / 08 / 2001$ & $22: 00$ & 31.7 & -12 & 18.2 & 9.07 & 316659 & 46.5 & 599 & 21.8 & 15.08 & 7 & -105 & 1536 & 132 & 18.99 & -7.13 & 60.19 \\
\hline 39 & $26 / 09 / 2001$ & $2: 00$ & 22.1 & -10 & 22 & 4.33 & $1.00 \mathrm{E}+06$ & 40.4 & 677 & 37.8 & 5.24 & 7.3 & -102 & 1753 & 154 & 14.96 & -6.97 & 33.06 \\
\hline 40 & $01 / 10 / 2001$ & $9: 00$ & 17.2 & -10 & 8.5 & 6.22 & 323152 & 18.9 & 622 & 9.81 & 1.04 & 6 & -148 & 949 & 80 & 10.69 & -6.4 & 18.4 \\
\hline 41 & $03 / 10 / 2001$ & $15: 00$ & 22.9 & -22 & 18.7 & 10.95 & 21321 & 13.7 & 573 & 6.7 & 16.13 & 7 & -166 & 1176 & 132 & 13.12 & -12.49 & 30.04 \\
\hline 42 & $21 / 10 / 2001$ & $22: 00$ & 26.1 & -17 & 6.6 & 10.64 & 529177 & 24.8 & 676 & 26.9 & 2.22 & 7.7 & -187 & 1307 & 179 & 17.64 & -11.42 & 46.04 \\
\hline 43 & $28 / 10 / 2001$ & $12: 00$ & 18.4 & -9.9 & 7.7 & 7.28 & 191696 & 11 & 502 & 5.29 & 16.43 & 6.7 & -157 & 950 & 111 & 9.23 & -4.97 & 16.99 \\
\hline 44 & $01 / 11 / 2001$ & 11:00 & 13.9 & -12 & 23.2 & 5.03 & 103199 & 23.4 & 387 & 8.42 & 10.26 & 5 & -106 & 676 & 48 & 5.38 & -4.76 & 7.48 \\
\hline 45 & $06 / 11 / 2001$ & $7: 00$ & 64.8 & -61 & 30.7 & 6.05 & 216397 & 42.6 & 729 & 14.4 & 22.28 & 8.7 & -292 & 1991 & 300 & 47.24 & -4.45 & 306.11 \\
\hline
\end{tabular}


UGC Approved Journal

IARJSET

ISSN (Online) 2393-8021

ISSN (Print) 2394-1588

International Advanced Research Journal in Science, Engineering and Technology ISO 3297:2007 Certified

Vol. 4, Issue 7, July 2017

\begin{tabular}{|c|c|c|c|c|c|c|c|c|c|c|c|c|c|c|c|c|c|c|}
\hline 46 & $24 / 11 / 2001$ & $17: 00$ & 49.3 & -33 & 8.5 & 23.44 & $2.00 \mathrm{E}+06$ & 43.9 & 1040 & 7 & 4.25 & 8.3 & -221 & 2006 & 236 & 51.27 & -3.43 & 252.77 \\
\hline 47 & $24 / 03 / 2002$ & $10: 00$ & 19.9 & -8.7 & 18 & 4.51 & 324757 & 15.3 & 568 & 5.36 & 2.64 & 6 & -100 & 1025 & 80 & 11.3 & -4.94 & 22.49 \\
\hline 48 & $18 / 04 / 2002$ & $8: 00$ & 27.2 & -16 & 12.3 & 9.36 & 396426 & 33.6 & 611 & 14.8 & 1.27 & 7.3 & -127 & 1356 & 154 & 16.62 & -9.65 & 45.2 \\
\hline 49 & $20 / 04 / 2002$ & 9:00 & 20.7 & -14 & 14.4 & 8.35 & 531934 & 12.1 & 669 & 8.73 & 1.95 & 7.3 & -149 & 1639 & 154 & 13.85 & -9.29 & 28.67 \\
\hline 50 & $11 / 05 / 2002$ & $20: 00$ & 17.6 & -15 & 35.4 & 7.23 & 262009 & 57.2 & 523 & 21.3 & 5.26 & 6.7 & -110 & 1287 & 111 & 9.2 & -7.95 & 16.2 \\
\hline 51 & $23 / 05 / 2002$ & 18:00 & 31.2 & -14 & 9.5 & 10.83 & $1.00 \mathrm{E}+06$ & 17.1 & 871 & 20.1 & 1.21 & 8.3 & -109 & 1480 & 236 & 27.18 & -12.28 & 84.79 \\
\hline 52 & $02 / 08 / 2002$ & 6:00 & 14.8 & -13 & 6.7 & 6 & 311710 & 13.8 & 524 & 7.53 & 1.81 & 6 & -102 & 1125 & 80 & 7.76 & -6.86 & 11.48 \\
\hline 53 & $21 / 08 / 2002$ & $7: 00$ & 12.6 & -8.9 & 14.6 & 4.2 & 143166 & 12.9 & 495 & 5.08 & 2.96 & 6.3 & -106 & 1146 & 94 & 6.24 & -4.4 & 7.86 \\
\hline 54 & $08 / 09 / 2002$ & $1: 00$ & 22.4 & -22 & 16.1 & 11.7 & 327861 & 17.5 & 550 & 7.76 & 1.61 & 7.3 & -181 & 1413 & 154 & 12.32 & -12.26 & 27.59 \\
\hline 55 & $01 / 10 / 2002$ & 17:00 & 24.1 & -14 & 8.8 & 9 & 246987 & 42.6 & 505 & 10.7 & 3.01 & 7.3 & -176 & 1088 & 154 & 12.17 & -69.18 & 29.33 \\
\hline 56 & $04 / 10 / 2002$ & $9: 00$ & 14 & -11 & 22.9 & 4.71 & 151059 & 11.7 & 516 & 5.66 & 2.5 & 7.3 & -146 & 1159 & 154 & 7.22 & -5.68 & 10.11 \\
\hline 57 & $30 / 05 / 2003$ & $0: 00$ & 27.8 & -12 & 8.8 & 9.61 & $1.00 \mathrm{E}+06$ & 36 & 806 & 39.1 & 4.72 & 8.3 & -144 & 1957 & 236 & 22.41 & -9.35 & 62.29 \\
\hline 58 & $18 / 06 / 2003$ & 10:00 & 19.7 & -17 & 22.9 & 8.53 & 361248 & 10.8 & 626 & 6.18 & 1.62 & 6.7 & -141 & 1297 & 111 & 12.33 & -10.77 & 24.29 \\
\hline 59 & $18 / 08 / 2003$ & 16:00 & 21.9 & -17 & 9.7 & 7.88 & 236723 & 17.6 & 530 & 9.18 & 4.65 & 7.3 & -148 & 1354 & 154 & 11.61 & -9.22 & 25.42 \\
\hline 60 & $30 / 10 / 2003$ & $1: 00$ & 46.7 & -25 & 10.1 & 26.22 & 843705 & 4.1 & 1084 & 9.56 & 0.23 & 9 & -353 & 2241 & 400 & 50.62 & -26.67 & 236.4 \\
\hline 61 & $30 / 10 / 2003$ & $23: 00$ & 36.7 & -28 & 23.5 & 2.52 & 144508 & 6 & 1189 & 13.8 & 2.45 & 9 & -389 & 2147 & 400 & 43.64 & -33.52 & 160.14 \\
\hline 62 & $20 / 11 / 2003$ & $21: 00$ & 55.7 & -46 & 28.9 & 31.25 & 534783 & 20.5 & 703 & 16.3 & 2.14 & 8.7 & -442 & 1698 & 300 & 39.16 & -32.27 & 218.1 \\
\hline 63 & $22 / 01 / 2004$ & $14: 00$ & 24.4 & -15 & 18.3 & 9.49 & 585976 & 17.7 & 666 & 15.2 & 6.2 & 7 & -149 & 1152 & 132 & 16.25 & -9.99 & 39.65 \\
\hline 64 & $04 / 04 / 2004$ & $1: 00$ & 18.2 & -9.3 & 8.6 & 3.96 & 121421 & 22.8 & 515 & 9.95 & 3.25 & 6.3 & -112 & 1130 & 94 & 9.37 & -4.79 & 17.06 \\
\hline 65 & $23 / 07 / 2004$ & $3: 00$ & 17.6 & -16 & 8.7 & 10.25 & 581133 & 15.5 & 672 & 6.33 & 2.2 & 7 & -101 & 1455 & 132 & 11.83 & -10.42 & 20.82 \\
\hline 66 & $25 / 07 / 2004$ & $12: 00$ & 23 & -4.9 & 16.3 & 10.58 & 548972 & 16.5 & 692 & 12.3 & 1.54 & 8 & -148 & 1635 & 207 & 15.92 & -15.15 & 36.61 \\
\hline 67 & $27 / 07 / 2004$ & 14:00 & 25.8 & -18 & 9.1 & 17.76 & $2.00 \mathrm{E}+06$ & 9 & 1027 & 19 & 2.16 & 8.7 & -197 & 1940 & 300 & 26.5 & -18.18 & 68.36 \\
\hline 68 & $30 / 08 / 2004$ & 23:00 & 14.9 & -14 & 4.9 & 5.72 & 307443 & 19.8 & 518 & 5.91 & 4.79 & 7 & -126 & 991 & 132 & 7.72 & -7.4 & 11.5 \\
\hline 69 & $08 / 11 / 2004$ & $7: 00$ & 45.8 & -45 & 29.1 & 29.45 & 808505 & 64.5 & 730 & 32.8 & 5.45 & 8.7 & -373 & 1753 & 300 & 33.43 & -32.56 & 153.13 \\
\hline 70 & $10 / 11 / 2004$ & 10:00 & 39 & -28 & 20.4 & 17.98 & 935977 & 21 & 809 & 25.2 & 10.28 & 8.7 & -289 & 1912 & 300 & 31.55 & -22.49 & 123.05 \\
\hline 71 & $12 / 11 / 2004$ & 11:00 & 12.4 & -5.4 & 9.7 & 2.58 & 424844 & 19.1 & 672 & 14.4 & 7.18 & 4.7 & -109 & 818 & 39 & 8.33 & -3.63 & 10.33 \\
\hline 72 & $20 / 01 / 2005$ & $7: 00$ & 26.6 & -6.9 & 23.8 & 5.71 & 664599 & 33.6 & 950 & 60.7 & 3.88 & 8 & -105 & 2111 & 207 & 25.27 & -6.55 & 67.21 \\
\hline 73 & $15 / 05 / 2005$ & $9: 00$ & 54.1 & -41 & 16.5 & 34.01 & 891191 & 19.1 & 959 & 25.5 & 6.23 & 8.3 & -263 & 1184 & 236 & 51.88 & -39.51 & 280.68 \\
\hline 74 & $20 / 05 / 2005$ & $9: 00$ & 14.9 & -9.7 & 7.2 & 4.3 & 165803 & 22.1 & 478 & 9.58 & 4.76 & 5 & -103 & 1050 & 48 & 7.12 & -4.64 & 10.61 \\
\hline 75 & $30 / 05 / 2005$ & 14:00 & 18.7 & -16 & 8.7 & 7.41 & 268294 & 17 & 541 & 8.76 & 36.48 & 7.7 & -138 & 1357 & 179 & 10.12 & -8.66 & 18.91 \\
\hline 76 & $13 / 06 / 2005$ & $1: 00$ & 23.4 & -17 & 15 & 8.4 & 283518 & 48.7 & 553 & 12.2 & 7.94 & 7.3 & -106 & 1561 & 154 & 12.94 & -9.35 & 30.28 \\
\hline 77 & $24 / 08 / 2005$ & 12:00 & 43.2 & -41 & 35.3 & 23.6 & 483718 & 29.6 & 720 & 22.8 & 3.29 & 8.7 & -216 & 2227 & 300 & 31.1 & -29.45 & 134.37 \\
\hline 78 & $11 / 09 / 2005$ & $11: 00$ & 18.1 & -6.4 & 9.8 & 5.47 & $1.00 \mathrm{E}+06$ & 7.6 & 1059 & 9.2 & 2.01 & 7.7 & -147 & 1383 & 179 & 19.17 & -6.78 & 34.69 \\
\hline 79 & $14 / 04 / 2006$ & 10:00 & 19.5 & -12 & 7 & 7.3 & 322684 & 16.5 & 676 & 8.1 & 3.93 & 7 & -111 & 1437 & 132 & 13.18 & -8.25 & 25.7 \\
\hline 80 & $15 / 12 / 2006$ & 8:00 & 17.8 & -16 & 11.1 & 12.39 & 915012 & 10.5 & 896 & 13.5 & 7.35 & 8.3 & -146 & 1616 & 236 & 15.95 & -13.98 & 28.39 \\
\hline 81 & $06 / 08 / 2011$ & $3: 00$ & 12.1 & -0.5 & 2.4 & 0.28 & 485385 & 8.6 & 567 & 5.29 & 1.48 & 5 & -115 & 740 & 48 & 6.86 & -0.29 & 8.3 \\
\hline 82 & $26 / 09 / 2011$ & $23: 00$ & 12.1 & -3.8 & 2.4 & 2.5 & 143172 & 2.7 & 657 & 2.13 & 0.21 & 5.3 & -118 & 393 & 56 & 7.95 & -2.49 & 9.62 \\
\hline 83 & $24 / 10 / 2011$ & $1: 00$ & 21.3 & -8 & 1.8 & 4.13 & 67094 & 12 & 516 & 6.25 & 0.22 & 7.3 & -147 & 483 & 154 & 10.99 & -4.13 & 23.41 \\
\hline 84 & $09 / 03 / 2012$ & $8: 00$ & 16 & -11 & 5.3 & 7.56 & 22550 & 3.2 & 683 & 3.71 & 0.08 & 8 & -131 & 1138 & 207 & 10.93 & -7.24 & 17.48 \\
\hline 85 & $24 / 04 / 2012$ & $4: 00$ & 10.6 & -7.3 & 1.6 & 3.05 & 177017 & 6.3 & 713 & 2.18 & 0.71 & 5.7 & -108 & 606 & 67 & 7.56 & -5.2 & 8.01 \\
\hline 86 & $15 / 07 / 2012$ & 18:00 & 21.9 & -16 & 4.2 & 8.85 & 31626 & 3.6 & 418 & 2.37 & 0.05 & 7 & -127 & 951 & 132 & 9.15 & -6.77 & 20.05 \\
\hline 87 & $16 / 07 / 2012$ & $7: 00$ & 14.3 & -13 & 3.2 & 5.86 & 73047 & 0.4 & 546 & 0.22 & 0.02 & 4.7 & -102 & 745 & 39 & 7.81 & -6.82 & 11.17 \\
\hline 88 & $01 / 10 / 2012$ & $4: 00$ & 20.7 & -7.8 & 2.3 & 3.07 & 515558 & 7.4 & 469 & 2.1 & 0.13 & 5.7 & -119 & 851 & 67 & 9.71 & -3.66 & 20.09 \\
\hline 89 & $09 / 10 / 2012$ & $8: 00$ & 15.7 & -6.6 & 3.1 & 2.91 & 43996 & 1.6 & 394 & 0.58 & 0.22 & 5.7 & -105 & 625 & 67 & 6.18 & -2.6 & 9.71 \\
\hline 90 & $14 / 11 / 2012$ & $7: 00$ & 17 & -15 & 3.5 & 5.96 & 158370 & 6 & 441 & 1.65 & 0.22 & 5.7 & -108 & 627 & 67 & 7.49 & -6.62 & 12.74 \\
\hline 91 & $17 / 03 / 2013$ & $20: 00$ & 12.4 & -5.6 & 5.2 & 3.42 & 92491 & 3.6 & 610 & 2.65 & 0.22 & 6.7 & -132 & 836 & 111 & 7.56 & -3.42 & 9.38 \\
\hline 92 & $01 / 06 / 2013$ & $8: 00$ & 19.6 & -4.5 & 1.6 & 2.25 & 361834 & 10.4 & 501 & 5.34 & 0.55 & 6.3 & -119 & 807 & 94 & 9.82 & -2.25 & 19.25 \\
\hline 93 & $19 / 02 / 2014$ & $8: 00$ & 18.4 & -6.7 & 4.4 & 3.05 & 64187 & 9.7 & 455 & 3.64 & 0.23 & 6.3 & -116 & 520 & 94 & 8.37 & -3.05 & 15.4 \\
\hline 94 & $17 / 03 / 2015$ & $22: 00$ & 30.2 & -4.1 & 5.8 & 10.57 & 912227 & 38.5 & 609 & 20.52 & 2.44 & 7.7 & -223 & 1570 & 179 & 18.3 & -2.49 & 55.54 \\
\hline 95 & $01 / 01 / 2016$ & $0: 00$ & 15 & -14 & 7.1 & 6.48 & 37966 & 1.8 & 463 & 0.58 & 0.06 & 6 & -110 & 0 & 80 & 6.95 & -6.48 & 10.42 \\
\hline \multicolumn{19}{|c|}{ CIR Driven } \\
\hline 1 & $23 / 10 / 1996$ & $5: 00$ & 12.8 & -10 & 7.7 & 6.48 & 347442 & 13 & 671 & 6.38 & 4.08 & 7.3 & -105 & 0 & 154 & 8.59 & -6.84 & 10.99 \\
\hline 2 & $10 / 03 / 1996$ & $21: 00$ & 21.6 & -13 & 19.4 & 7.47 & 362284 & 51.9 & 553 & 12.7 & 3.73 & 7.3 & -116 & 881 & 154 & 11.99 & -7.13 & 25.8 \\
\hline 3 & $24 / 05 / 2000$ & $9: 00$ & 30.8 & -24 & 26.6 & 12 & 468151 & 30.8 & 684 & 28 & 15.04 & 8 & -147 & 1336 & 207 & 21.07 & -16.48 & 64.89 \\
\hline 4 & $04 / 09 / 2002$ & $6: 00$ & 18.6 & -18 & 10.9 & 6.81 & 257857 & 18.4 & 461 & 5.09 & 2.08 & 6.3 & -109 & 884 & 94 & 8.57 & -8.29 & 15.95 \\
\hline 5 & $07 / 10 / 2002$ & $8: 00$ & 12.8 & -6 & 8.1 & 3.6 & 165988 & 11.5 & 511 & 3.36 & 2.34 & 6 & -115 & 1067 & 80 & 6.54 & -3.07 & 8.37 \\
\hline 6 & $14 / 10 / 2002$ & 14:00 & 17.3 & -16 & 11.5 & 4.62 & 502949 & 34.9 & 611 & 7.19 & 2.48 & 4.7 & -100 & 1044 & 39 & 10.57 & -9.78 & 18.29 \\
\hline 7 & $21 / 11 / 2002$ & $11: 00$ & 29.9 & -12 & 12.6 & 5.46 & 503038 & 52.6 & 727 & 24.1 & 3.64 & 6.7 & -128 & 1013 & 111 & 21.74 & -8.87 & 64.99 \\
\hline 8 & $12 / 07 / 2003$ & $6: 00$ & 15.1 & -14 & 8.8 & 7.77 & 529872 & 19.5 & 674 & 6.04 & 2.25 & 6.7 & -105 & 1248 & 111 & 10.18 & -9.09 & 15.37 \\
\hline 9 & $11 / 02 / 2004$ & 18:00 & 21.1 & -12 & 14.1 & 5.31 & 856405 & 21.5 & 735 & 6.71 & 1.96 & 6.3 & -109 & 994 & 94 & 15.51 & -9.11 & 32.72 \\
\hline 10 & $18 / 01 / 2005$ & 9:00 & 32.4 & -17 & 14.3 & 14.83 & $1.00 \mathrm{E}+06$ & 11.3 & 997 & 13.6 & 3.18 & 7.7 & -121 & 2136 & 179 & 32.3 & -16.75 & 104.66 \\
\hline 11 & $08 / 05 / 2005$ & 19:00 & 15.3 & -12 & 13.2 & 10.45 & 845807 & 47 & 821 & 14.7 & 3.88 & 8.3 & -127 & 1505 & 236 & 12.56 & -10.18 & 19.22 \\
\hline 12 & $31 / 08 / 2005$ & 20:00 & 17.3 & -17 & 11.2 & 6.11 & 648336 & 34.7 & 743 & 11.7 & 8.49 & 7 & -131 & 1162 & 132 & 12.85 & -12.56 & 22.24 \\
\hline 13 & $17 / 03 / 2015$ & 19:00 & 16 & -13 & 11.2 & 7.32 & 86307 & 10.6 & 576 & $* * *$ & 0.37 & 7.3 & -165 & 978 & 154 & 9.21 & -7.31 & 14.75 \\
\hline 14 & $23 / 06 / 2015$ & 12:00 & 5.9 & -0.9 & 12.1 & 4.57 & 729009 & 3.7 & 578 & $* * *$ & 3.79 & 5.7 & -138 & 1386 & 67 & 3.41 & -0.52 & 20.12 \\
\hline
\end{tabular}

Table 2. Correlation coefficient between various interplanetary parameters (IP) and geomagnetic Indices (GI) during the rising phase, maximum phase,

\begin{tabular}{|l|l|l|l|l|l|l|l|l|l|l|l|l|l|l|l|l|}
\hline \multicolumn{10}{|c|}{ Decay Phase of cycle 23 \& 24 and total period } \\
\hline IP/GI
\end{tabular}


UGC Approved Journal

\begin{tabular}{|l|l|l|l|l|l|l|l|l|l|l|l|l|l|l|l|l|}
\hline $\mathrm{E}$ & -0.74 & 0.76 & 0.81 & 0.37 & -0.74 & 0.64 & 0.77 & 0.6 & -0.7 & 0.64 & 0.63 & 0.37 & -0.73 & 0.55 & 0.73 & 0.54 \\
\hline $\mathrm{T}$ & -0.42 & 0.48 & 0.54 & 0.36 & -0.45 & 0.52 & 0.6 & 0.62 & -0.22 & 0.47 & 0.43 & 0.47 & -0.34 & 0.51 & 0.54 & 0.54 \\
\hline $\mathrm{D}$ & -0.16 & 0.24 & 0.18 & 0.09 & -0.28 & 0.36 & 0.33 & 0.48 & -0.11 & 0.17 & 0.08 & 0.26 & 0.13 & 0.24 & 0.17 & 0.23 \\
\hline $\mathrm{V}$ & -0.58 & 0.57 & 0.65 & 0.24 & -0.58 & 0.65 & 0.7 & 0.61 & -0.49 & 0.66 & 0.74 & 0.63 & -0.53 & 0.65 & 0.72 & 0.63 \\
\hline $\mathrm{P}$ & 0.09 & 0.04 & 0.01 & 0.07 & -0.53 & 0.63 & 0.63 & 0.68 & -0.19 & 0.44 & 0.38 & 0.54 & -0.41 & 0.57 & 0.56 & 0.55 \\
\hline$\beta$ & 0.24 & -0.18 & -0.23 & -0.1 & -0.17 & 0.2 & 0.17 & 0.25 & 0.11 & 0.01 & -0.04 & -0.01 & 0.02 & 0.08 & 0.04 & 0.12 \\
\hline $\mathrm{BV}$ & -0.74 & 0.73 & 0.78 & 0.29 & -0.62 & 0.56 & 0.66 & 0.62 & -0.82 & 0.77 & 0.86 & 0.61 & -0.75 & 0.74 & 0.83 & 0.68 \\
\hline BzV & 0.63 & -0.64 & -0.62 & -0.44 & 0.53 & -0.44 & -0.52 & -0.3 & 0.83 & -0.72 & -0.8 & -0.48 & 0.73 & -0.66 & 0.77 & 0.58 \\
\hline $\mathrm{B}^{2} \mathrm{~V}$ & -0.66 & 0.62 & 0.68 & 0.24 & -0.61 & 0.53 & 0.55 & 0.61 & -0.84 & 0.66 & 0.76 & 0.49 & 0.77 & 0.66 & 0.77 & 0.68 \\
\hline
\end{tabular}

Table.3. Average (AV),Median(Med) and standard deviations (SD) of various geomagnetic indices(GIs), interplanetry field parameters, plasma parameters and their prouduct functions during the rising, maximum and decay phases of cycle $23 \& 24$ as well as the total period 1996-2016.

\begin{tabular}{|c|c|c|c|c|c|c|c|c|c|c|c|c|}
\hline & \multicolumn{3}{|c|}{$\begin{array}{l}\text { Rising Phase } \\
\text { (cycle 23 \&24) }\end{array}$} & \multicolumn{3}{|c|}{$\begin{array}{l}\text { Maximum Phase } \\
\text { (cycle } 23 \text { \&24) }\end{array}$} & \multicolumn{3}{|c|}{$\begin{array}{l}\text { Decay Phase } \\
\text { (cycle } 23 \text { \&24) }\end{array}$} & \multicolumn{3}{|c|}{$\begin{array}{l}\text { Total Phase } \\
(1996-2016)\end{array}$} \\
\hline & AV & Med & SD & AV & Med & SD & $\mathrm{AV}$ & Med & SD & AV & Med & SD \\
\hline Dst(nT) & -133.47 & -117 & 35.98 & -150.5 & -127 & 63.43 & -185.42 & -146.5 & 97.79 & -154 & -128 & 70.29 \\
\hline $\mathrm{Kp}(\mathrm{nT})$ & 6.89 & 6.85 & 0.94 & 6.9 & 6.85 & 1.05 & 7.49 & 7.7 & 1.17 & 7.06 & 7 & 1.07 \\
\hline $\operatorname{ap}(n T)$ & 135.65 & 121.5 & 60.25 & 139.26 & 121.5 & 78.55 & 192.42 & 179 & 97.9 & 152.6 & 132 & 81.99 \\
\hline A.E(nT) & 850 & 949.5 & 452.81 & 1192.8 & 1129 & 376.2 & 1476 & 1446 & 490.46 & 1228 & 1153 & 441.8 \\
\hline $\mathrm{Bt}(\mathrm{nT})$ & 21.16 & 21 & 7.52 & 23.42 & 20.3 & 10.94 & 26.17 & 22.45 & 13.02 & 23.44 & 20.7 & 10.74 \\
\hline $\mathrm{Bz}(\mathrm{nT})$ & -14.12 & -13 & 6.41 & -16.58 & -13.5 & 10.87 & -17.8 & -15.5 & 12.45 & -16.2 & -13.5 & 10.2 \\
\hline$\sigma \mathrm{B}(\mathrm{nT})$ & 10.16 & 10.7 & 7.28 & 12.87 & 11.9 & 9.98 & 13.3 & 9.75 & 8.99 & 13.4 & 11 & 14.52 \\
\hline $\mathrm{E}(\mathrm{mV} / \mathrm{m})$ & 7.99 & 6.59 & 4.85 & 9.1 & 7.05 & 8.05 & 11.68 & 8.46 & 9.17 & 9.49 & 7.32 & 7.64 \\
\hline $\mathrm{T}\left({ }^{\circ} \mathrm{K}\right)$ & 415321 & 336190 & 396380 & 397790 & 314180 & 409760 & 578410 & 541880 & 412900 & $5.00 \mathrm{E}+05$ & 347442 & $4.00 \mathrm{E}+05$ \\
\hline $\mathrm{D}\left(\mathrm{cm}^{-3}\right)$ & 26.34 & 24.4 & 15.36 & 22.71 & 19.75 & 14.15 & 20.01 & 17.65 & 14.06 & 23.06 & 20.5 & 14.42 \\
\hline $\mathrm{V}(\mathrm{km} / \mathrm{s})$ & 589.31 & 560 & 120.84 & 607.98 & 578.5 & 158.64 & 724.89 & 674 & 201.23 & 642.1 & 609 & 171.9 \\
\hline $\mathrm{P}(\mathrm{nPa})$ & 53.61 & 11 & 211.92 & 13.62 & 9.84 & 12.97 & 15.23 & 12.25 & 12.85 & 23.87 & 11.05 & 104.9 \\
\hline$\beta$ (beta) & 4.97 & 3.91 & 3.88 & 5.03 & 2.8 & 6.84 & 5.04 & 3.83 & 6.63 & 4.97 & 3.33 & 6.003 \\
\hline $\mathrm{BV} \times 10^{3}(\mathrm{nTkm} / \mathrm{s})$ & 33.89 & 23.65 & 28.09 & 16.48 & 11.34 & 13.09 & 20.18 & 15.93 & 13.55 & 16.56 & 12.08 & 12.02 \\
\hline $\mathrm{BzV} \times 10^{3}(\mathrm{nTkm} / \mathrm{S})$ & -8.51 & -7.27 & 4.79 & -10.81 & -7.18 & 12.09 & -13.94 & -9.35 & 10.91 & -11.2 & -8.1 & 10.29 \\
\hline $\mathrm{B}^{2} \mathrm{~V} \times 10^{4}\left((\mathrm{nT})^{2} \mathrm{~km} / \mathrm{s}\right)$ & 12.86 & 11.18 & 6.51 & 42.25 & 22.32 & 57.73 & 69.24 & 32.48 & 76.09 & 46.95 & 24.435 & 58.37 \\
\hline
\end{tabular}
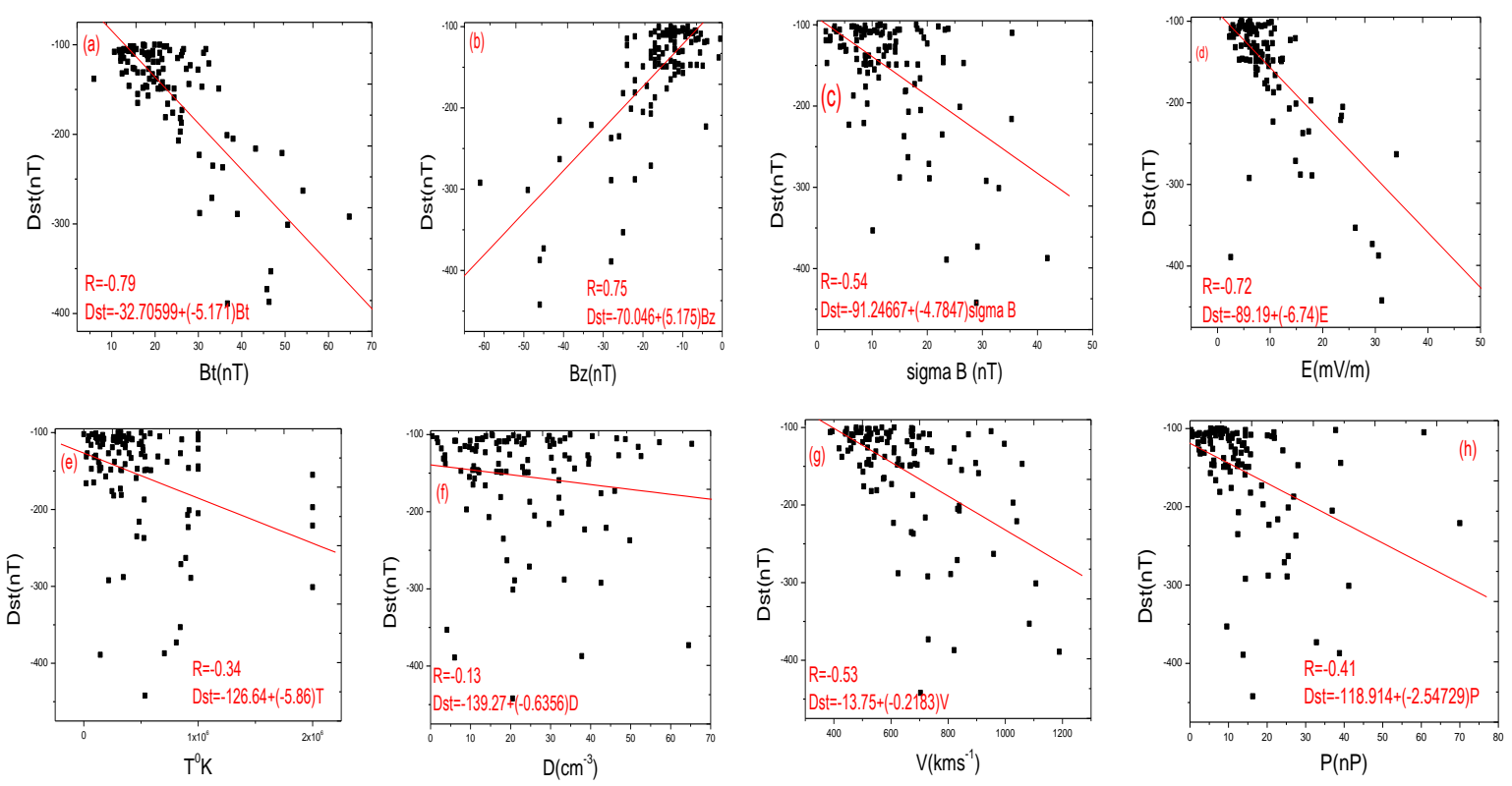


\section{UGC Approved Journal}
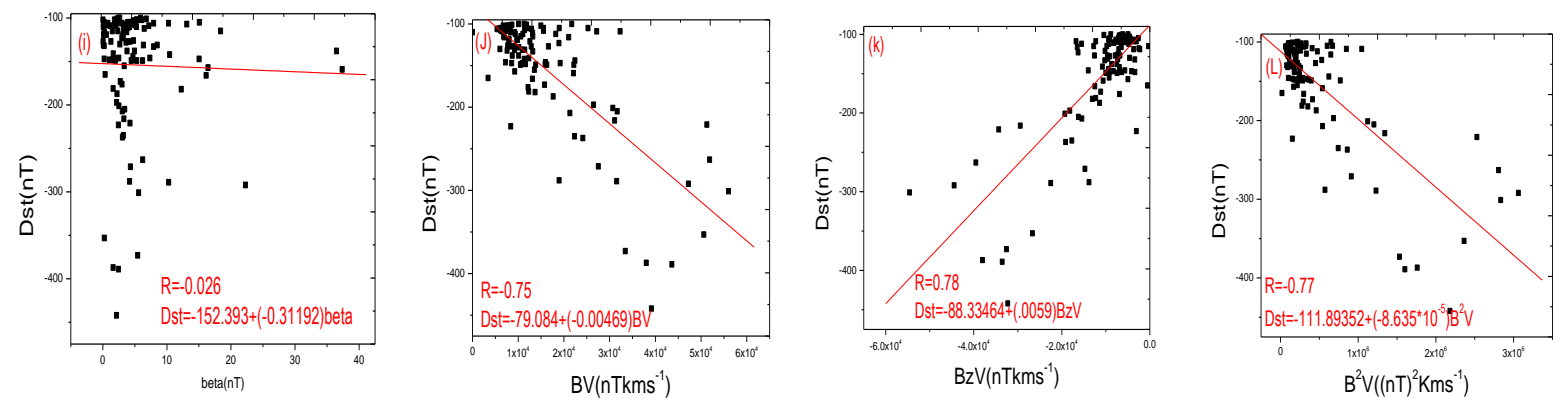

Fig:1. Relations of Dst with interplanetary fields( Bt,Bz,Sigma B, E), plasma parameters( T, P,V, D, Beta $(\beta))$ and there product $\left(\mathrm{BtV}, \mathrm{BzV}, \mathrm{B}^{2} \mathrm{~V}\right)$.
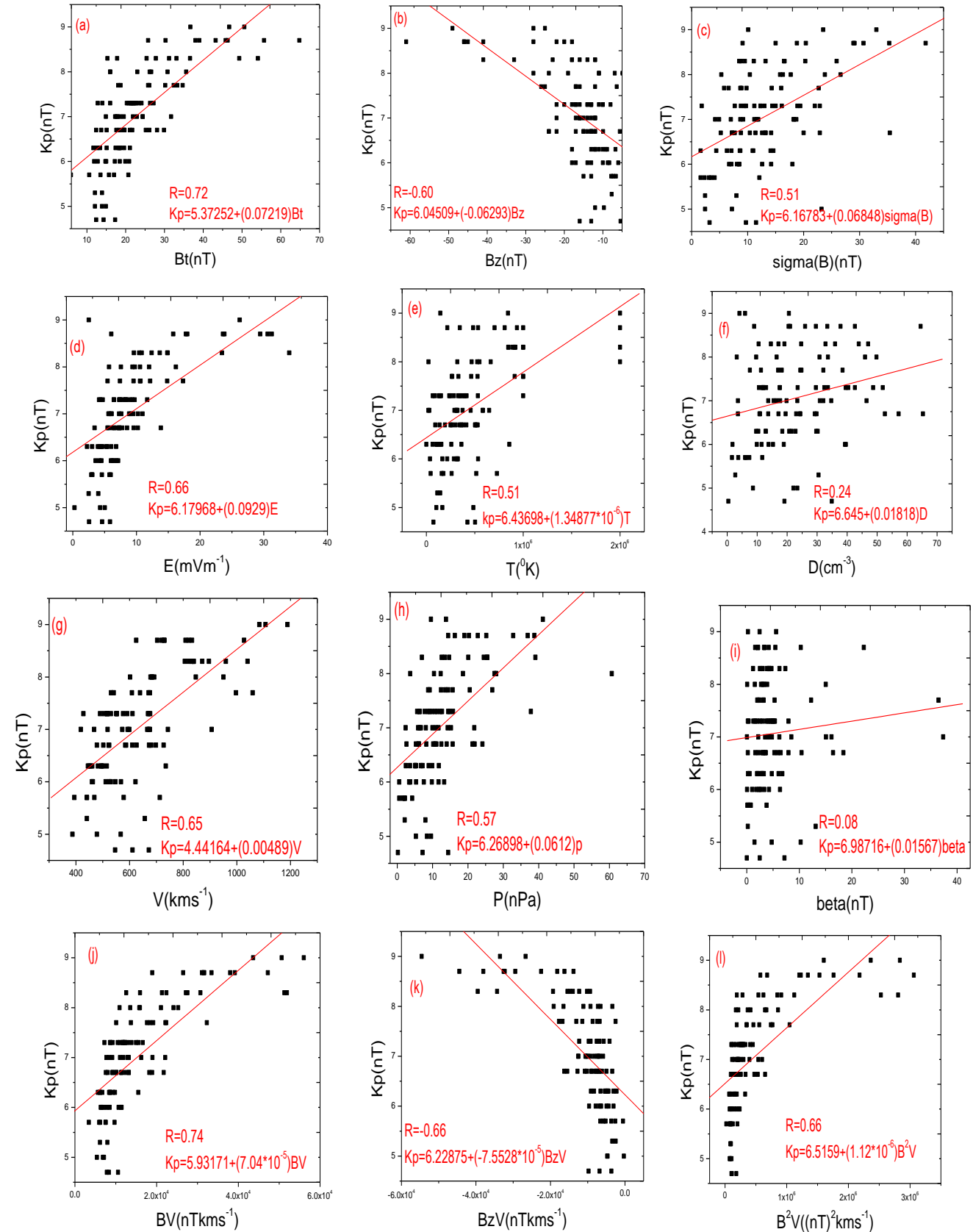

Fig:2. Relations of Kp with interplanetary fields( Bt,Bz,Sigma B, E), plasma parameters( T, P,V, D, Beta $(\beta))$ and there product $(\mathrm{BtV}, \mathrm{BzV}, \mathrm{B} 2 \mathrm{~V})$. 
UGC Approved Journal
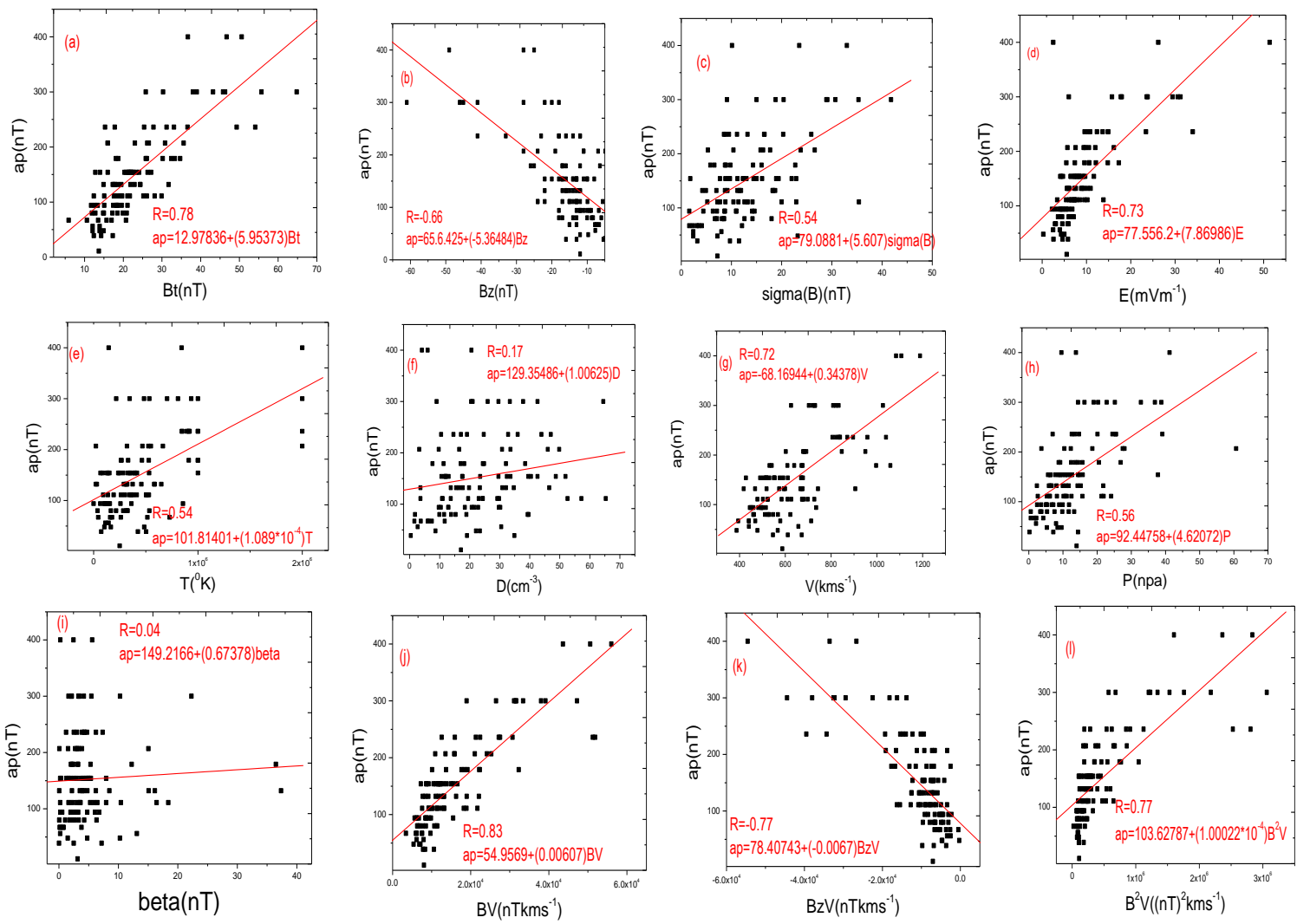

Fig:3. Relations of ap with interplanetary fields( Bt,Bz,Sigma B, E), plasma parameters ( T, P,V, D, Beta( $\beta)$ ) and there product $\left(\mathrm{BtV}, \mathrm{BzV}, \mathrm{B}^{2} \mathrm{~V}\right)$.
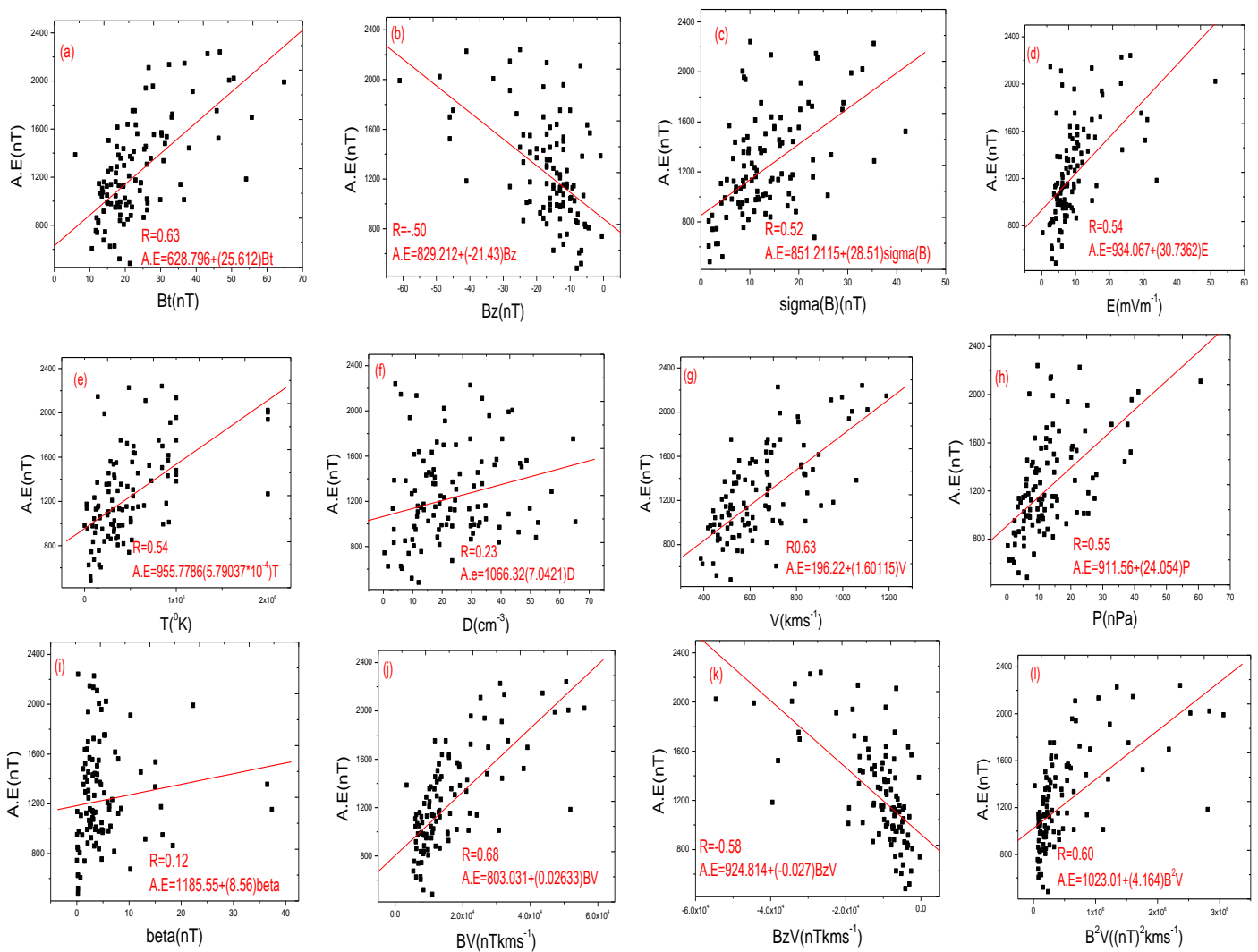

Fig:4. Relations of A.E with interplanetary fields (Bt, Bz, Sigma B, E), plasma parameters( T, P,V, D, Beta( $(\beta))$ and there product $\left(\mathrm{BtV}, \mathrm{BzV}, \mathrm{B}^{2} \mathrm{~V}\right)$. 